\title{
Fusion of ferredoxin and cytochrome P450 enables direct light-driven biosynthesis
}

Mellor, Silas Busck; Nielsen, Agnieszka Janina Zygadlo; Burow, Meike; Motawie, Mohammed Saddik; Jakubauskas, Dainius; Møller, Birger Lindberg; Jensen, Poul Erik

Published in:

ACS chemical biology

DOI:

10.1021/acschembio.6b00190

Publication date:

2016

Document version

Publisher's PDF, also known as Version of record

Citation for published version (APA):

Mellor, S. B., Nielsen, A. J. Z., Burow, M., Motawie, M. S., Jakubauskas, D., Møller, B. L., \& Jensen, P. E. (2016). Fusion of ferredoxin and cytochrome P450 enables direct light-driven biosynthesis. ACS chemical biology, 11(7), 1862-1869. https://doi.org/10.1021/acschembio.6b00190 


\title{
Fusion of Ferredoxin and Cytochrome P450 Enables Direct Light- Driven Biosynthesis
}

\author{
Silas Busck Mellor, ${ }^{\dagger, \S}$ Agnieszka Zygadlo Nielsen, ${ }^{\dagger, \S}$ Meike Burow, ${ }^{\dagger, \ddagger}$ Mohammed Saddik Motawia, ${ }^{\dagger, \S}$
} Dainius Jakubauskas, ${ }^{\dagger, \S}$ Birger Lindberg Møller, ${ }^{\dagger},, \|$ and Poul Erik Jensen*, ${ }^{\dagger}, \S, \|$

${ }^{\dagger}$ Copenhagen Plant Science Center, Department of Plant and Environmental Sciences, University of Copenhagen, Thorvaldsensvej 40, 1871 Frederiksberg C, Denmark

${ }^{\ddagger}$ DynaMo Center of Excellence, Department of Plant and Environmental Sciences, University of Copenhagen, Thorvaldsensvej 40, 1871 Frederiksberg C, Denmark

${ }^{\S}$ Center for Synthetic Biology "bioSYNergy", Thorvaldsensvej 40, 1871 Frederiksberg, Denmark

"Villum Research Center of Excellence ”Plant Plasticity”, University of Copenhagen, Thorvaldsensvej 40, 1871 Frederiksberg C, Denmark

\section{Supporting Information}

ABSTRACT: Cytochrome P450s (P450s) are key enzymes in the synthesis of bioactive natural products in plants. Efforts to harness these enzymes for in vitro and whole-cell production of natural products have been hampered by difficulties in expressing them heterologously in their active form, and their requirement for NADPH as a source of reducing power. We recently demonstrated targeting and insertion of plant P450s into the photosynthetic membrane and photosynthesis-driven, NADPHindependent P450 catalytic activity mediated by the electron carrier protein ferredoxin. Here, we report the fusion of ferredoxin with P450 CYP79A1 from the model plant Sorghum bicolor, which catalyzes the initial step in the pathway leading to biosynthesis of the cyanogenic glucoside

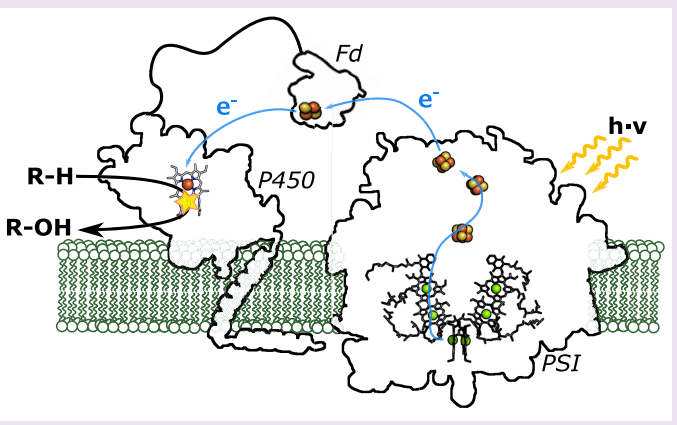
dhurrin. Fusion with ferredoxin allows CYP79A1 to obtain electrons for catalysis by interacting directly with photosystem I. Furthermore, electrons captured by the fused ferredoxin moiety are directed more effectively toward P450 catalytic activity, making the fusion better able to compete with endogenous electron sinks coupled to metabolic pathways. The P450-ferredoxin fusion enzyme obtains reducing power solely from its fused ferredoxin and outperforms unfused CYP79A1 in vivo. This demonstrates greatly enhanced electron transfer from photosystem I to CYP79A1 as a consequence of the fusion. The fusion strategy reported here therefore forms the basis for enhanced partitioning of photosynthetic reducing power toward P450-dependent biosynthesis of important natural products.

$\mathrm{W}$ ith the continued development of tools for engineering biosynthetic pathways into microorganisms, elucidation of routes leading to plant natural products of high value is attracting considerable interest. ${ }^{1-3}$ Cytochrome P450s (P450s) are key enzymes in specialized metabolism and are involved in the formation of terpenoids, alkaloids, cyanogenic glycosides, glucosinolates, and phenylpropanoids like flavonoids, coumarins, and stilbenes. They catalyze stereo- and regiospecific hydroxylations, epoxidations, and $\mathrm{C}-\mathrm{C}$ couplings that are often difficult to accomplish by chemical synthesis. In eukaryotes, P450s reside on the endoplasmic reticulum, and plant genomes often contain several hundred P450-encoding genes. ${ }^{4-6}$ Biotechnological production of many high-value specialized metabolites thus requires heterologous expression of one or more P450s in highly active form. The yeast Saccharomyces cerevisiae remains the favored host for introduction of plant P450-dependent pathways, ${ }^{1}$ but cyanobacteria are gaining in popularity and offer unparalleled sustainability because they too are photosynthetic organisms and therefore require minimal nutrient input. ${ }^{7}$ We recently showed that the thylakoid membranes of both cyanobacteria and plants, which host the photosynthetic apparatus, can also accommodate plant P450s. ${ }^{8-11}$

Two key issues encountered when engineering P450dependent pathways are the need for high-level functional expression of the $\mathrm{P} 450$ and the provision of sufficient reducing power to drive their catalytic cycle. ${ }^{12}$ This study addresses the latter. Many alternative P450 reductase systems and P450reductase fusions have been reported, but only a few have resulted in increased activity. ${ }^{13}$ Photosynthetic hosts offer several advantages for P450-dependent pathways: ${ }^{2,3}$ Plant $\mathrm{P} 450 \mathrm{~s}$ are functionally active when targeted to thylakoid membranes and benefit from the ample supply of electrons and molecular oxygen generated by photosynthetic electron trans-

Received: February 29, 2016

Accepted: April 27, 2016

Published: April 27, 2016 


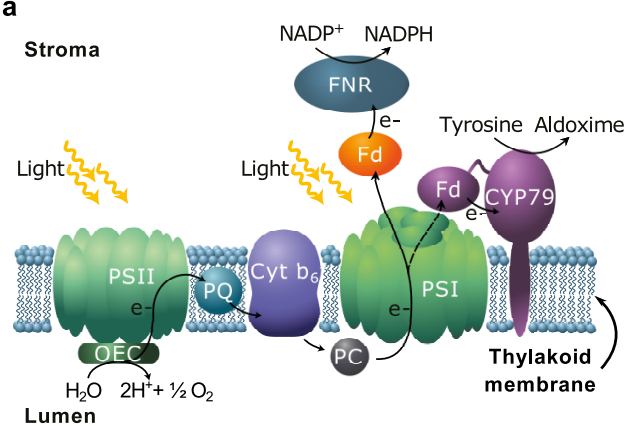

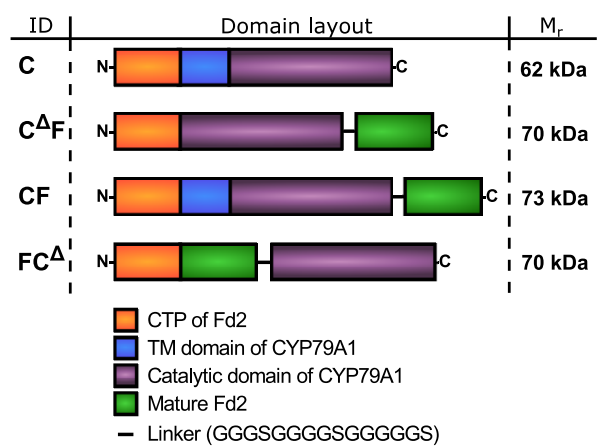

Figure 1. Overview of light-driven biosynthesis and fusion constructs used in this study. (a) Schematic illustration of the interfacing of photosynthetic electron transport with light-driven biosynthesis by fusing ferredoxin to CYP79A1 in the thylakoid membrane, thus directing electrons toward cytochrome P450-catalyzed hydroxylations. OEC, oxygen-evolving complex; PSII, photosystem II; PQ, plastoquinone pool; Cyt $\mathrm{b}_{6} \mathrm{f}$, cytochrome $b_{6} \mathrm{f}$; PC, plastocyanin; PSI, photosystem I; Fd, ferredoxin; FNR, ferredoxin:NADPH-reductase. (b) Design of gene constructs that fuse CYP79A1 from Sorghum bicolor to the major Fd of Arabidopsis thaliana, Fd2. ${ }^{35}$ All constructs included an N-terminal Fd chloroplast transit peptide (CTP), which targets them to the chloroplast. The mature Fd (F) was fused via a 15-residue linker either to the full-length CYP79A1 (C) or to its heme domain lacking the transmembrane $(\mathrm{TM})$ anchor $\left(\mathrm{C}^{\Delta}\right)$. ID, abbreviated construct names; $\mathrm{M}_{\mathrm{r}}$, calculated mass (not counting the transit peptide).

a

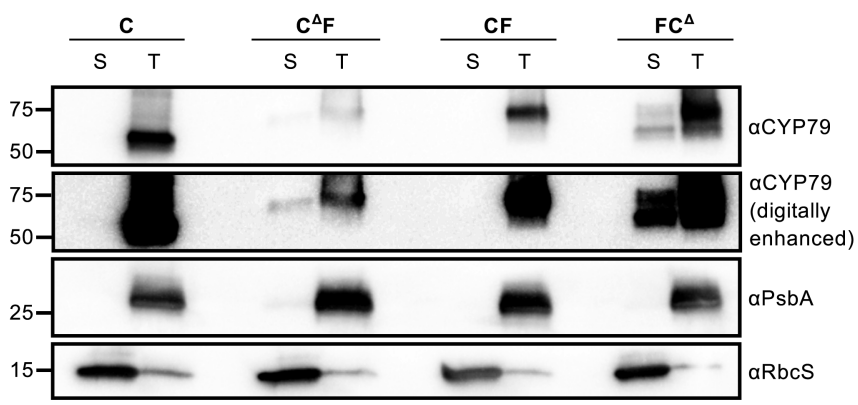

b

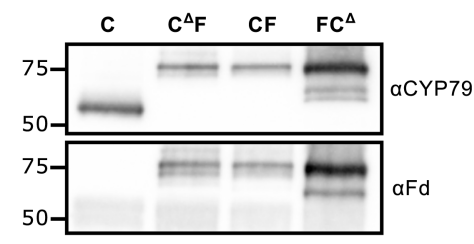

Figure 2. CYP79A1-ferredoxin fusion enzymes localized to thylakoid membranes of tobacco chloroplasts and containing both CYP79A1 and ferredoxin epitopes. (a) Immunoblot analysis of chloroplasts isolated from tobacco leaves 5 days after agroinfiltration and fractionated into stroma (S) and thylakoid (T) fractions. Membranes were probed with antibodies against either CYP79A1, the D1 protein of photosystem II (PsbA, a thylakoid marker), or a RuBisCo small subunit ( $\mathrm{RbcS}$, a stroma marker). Mobilities of protein standards of known mass (in $\mathrm{kDa}$ ) are indicated on the left. The immunoblot probed with the CYP79A1 antibody is shown as the original exposure and in a digitally enhanced version that emphasizes weak signals. (b) Immunoblot analysis of thylakoids isolated from tobacco expressing fusion constructs as in a, probed with antibodies against CYP79A1 or ferredoxin $(\mathrm{Fd})$.

port (Figure 1a). ${ }^{8,9}$ Cyanobacteria or plant-cell cultures may thus constitute a useful vehicle for environmentally contained, heterologous production of specialized plant metabolites of high value, such as structurally complex diterpenoids functionalized in P450-dependent reactions. ${ }^{14-18}$ However, competition from native electron sinks, such as the metabolic reactions involved in $\mathrm{CO}_{2}$ fixation and redox regulation processes, ${ }^{8}$ complicates the diversion of electrons from photosynthesis to non-native processes. In an effort to improve partitioning of electrons toward the P450, we have now designed and tested fusions that covalently connect the electron transfer protein ferredoxin $(\mathrm{Fd})$ to the well-characterized P450 CYP79A1 from Sorghum bicolor (Figure 1a).

In the current study, we expressed and targeted P450-Fd fusions to the chloroplast in Nicotiana benthamiana. One of the fusion constructs, which could acquire photosynthetic reducing power by direct electron transfer from photosystem I without the need for a dedicated reductase, was better able to compete with endogenous electron sinks and showed higher in vivo activity than the native enzyme. This study thus represents a proof of concept that P450-Fd fusions can interface directly with electron transfer from photosystem I and divert more photosynthetic reducing power to engineered metabolism.

\section{RESULTS}

Design of CYP79A1-Ferredoxin Fusion Proteins. The initial step in the biosynthetic pathway leading to the cyanogenic glucoside dhurrin in Sorghum bicolor ${ }^{19-21}$ is the conversion of L-tyrosine to (E)-p-hydroxyphenylacetaldoxime, which is catalyzed by CYP79A1. ${ }^{21-24}$ We chose CYP79A1 for our ferredoxin $(\mathrm{Fd})$ fusion studies because it is stable both in its native ER and in photosynthetic membranes ${ }^{8,9}$ and well characterized. $^{21,22,24}$ We designed three different fusion constructs - two C-terminal fusions named $\mathrm{C}^{\Delta} \mathrm{F}$ and $\mathrm{CF}$ and one N-terminal construct $\mathrm{FC}^{\Delta}$ (Figure $1 \mathrm{~b}$ ). A linker length of 15 amino acids was chosen based on the distances between the $\mathrm{C}$-terminus of CYP79A1 and the $\mathrm{N}$-terminus of $\mathrm{Fd}$ derived from docking models, the presence in CYP79A1 of four Cterminal residues with predicted random-coil secondary structure not resolved in crystal structures, ${ }^{25}$ and the estimated distance from the edge of photosystem I to its Fd binding site (Supporting Figure S1). A Gly/Ser-rich sequence was chosen to avoid secondary structure and maximize the ability of the $\mathrm{Fd}$ 
domain to transfer electrons to the heme of CYP79A1 and reduce susceptibility to proteases. $^{26}$ Because the native CYP79A1 (named C in Figure $1 b$ ) contains an $\mathrm{N}$-terminal transmembrane domain that normally anchors it to the ER membrane, N-terminal fusions to $\mathrm{Fd}$ were constructed with CYP79A1 lacking this domain, to avoid placing $\mathrm{Fd}$ and CYP79A1 domains on opposite sides of the thylakoid membrane. An additional C-terminal Fd fusion with the same truncated CYP79A1 variant was therefore assembled to control for differences due to a lack of the transmembrane anchor.

Chloroplast Sublocalization of Fusion Proteins. We used Agrobacterium-mediated infiltration of $N$. benthamiana leaves to generate plants that transiently expressed the CYP79A1 constructs. Subsequently, we fractionated intact chloroplasts prepared from infiltrated leaves and subjected each fraction to immunoblot analysis with an anti-CYP79 antibody to verify that the Fd transit peptide targeted fusion proteins to thylakoid membranes (Figure 2a). As expected, the fusions were found mainly in thylakoid fractions, though both $\mathrm{FC}^{\Delta}$ and $\mathrm{C}^{\Delta} \mathrm{F}$ were also detectable in the stromal fraction.

Immunoblot analyses performed with an antibody raised against ferredoxin showed clear signals for $\mathrm{C}^{\Delta} \mathrm{F}, \mathrm{CF}$, and $\mathrm{FC}^{\Delta}$ constructs, but not the unfused construct $C$ (Figure $2 b$ ), thus confirming that the translated fusion proteins contained both CYP79A1 and $\mathrm{Fd}$ domains. The $\mathrm{C}^{\Delta} \mathrm{F}, \mathrm{CF}$, and $\mathrm{FC}^{\Delta}$ proteins comprised $0.2-2.6 \%$ of total thylakoid protein, based on SDSPAGE densitometry (Supporting Figure S2c). The C protein could not be quantified by this approach because it comigrated with the abundant $\beta$-subunit of ATP synthase. A high pigment background (due to chlorophylls and carotenoids) in the isolated thylakoid membranes interfered with standard determination of concentrations of the $\mathrm{P} 450$ s by $\mathrm{CO}$ difference spectra, and we therefore measured their relative levels by immunoblot analysis (Supporting Figure S2a,b and Supporting Table S1).

Fusion with Fd Directs Electrons to CYP79A1 in the Absence of Soluble Fd. We next performed enzyme assays using thylakoid preparations containing $\mathrm{C}, \mathrm{C}^{\Delta} \mathrm{F}, \mathrm{CF}$, or $\mathrm{FC}^{\Delta}$ protein to investigate whether the $\mathrm{Fd}$ domain fused to $\mathrm{C}^{\Delta} \mathrm{F}$, $\mathrm{CF}$, and $\mathrm{FC}^{\Delta}$ mediated direct electron transfer from photosystem I to the heme of the CYP79A1 domain. In vitro assays were carried out to assess whether added soluble Fd was required for electron transfer between photosystem I and the P450s (Figure 3). Only the CF fusion protein harboring the full-length CYP79A1 protein was found to exhibit substantial activity in the absence of added soluble Fd. Direct electron transfer between Fd and CYP79A1 domains also operated in assays in which the $\mathrm{Fd}$ domain was reduced in a lightindependent manner by the enzyme ferredoxin:NADP ${ }^{+}$ reductase (FNR) in the presence of $\mathrm{NADPH}$ (Supporting Figure S3). Based on these findings, we focused subsequent experiments on characterizing the function of the CF enzyme.

Dependence of Activity on Soluble Ferredoxin. Because the activity of CF was unchanged by the addition of soluble Fd in our initial experiments, we performed light-driven activity assays with varying concentrations of soluble $\mathrm{Fd}$ to compare the fusion protein's dependence on soluble Fd with that of the unfused $C$ enzyme. Increasing the Fd concentration from 1 to $16.6 \mu \mathrm{M}$ caused only a modest 1.2 -fold increase in the activity of the CF enzyme, compared to a 4-fold increase and clear saturation in the activity of C (Figure 4a). To test whether the reduced activity of CF was due to suboptimal interaction with photosystem I, we performed activity assays

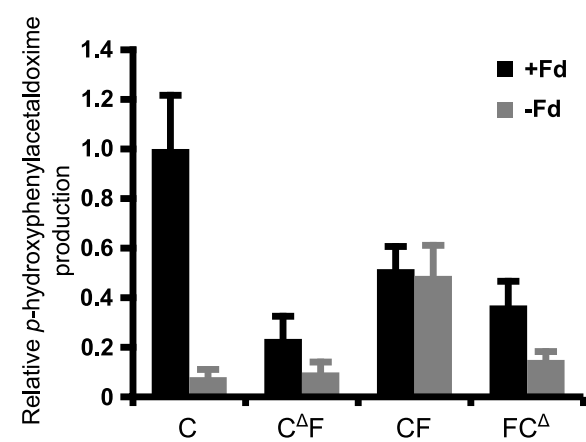

Figure 3. Comparison of in vitro light-driven enzyme activity from $N$. benthamiana thylakoids harboring CYP79A1 enzyme variants. Assays were carried out in the presence or absence of $8.3 \mu \mathrm{M}$ soluble $\mathrm{Fd}(+\mathrm{Fd}$ or $-\mathrm{Fd}$ ) as an electron carrier. Bars show average activities of four separate thylakoid preparations per construct, normalized to their relative protein levels as determined from immunoblotting experiments. Error bars indicate \pm SD of biological replicates.

with varying $\mathrm{Fd}$ concentrations under dark conditions, using a soluble electron donor system composed of NADPH and FNR (Figure $4 \mathrm{~b}$ ). As in the light-driven assays (Figure 4a), the $>15$ fold increase in Fd concentration only modestly enhanced $\mathrm{CF}$ activity (by 1.6-fold), while the activity of $\mathrm{C}$ increased 10 -fold and did not saturate (Figure $4 \mathrm{~b}$ ). Overall activity was 3-4 times lower for both $\mathrm{C}$ and $\mathrm{CF}$ in this assay compared to the lightdriven assay. To investigate the importance of thylakoid integrity for the activity of $\mathrm{C}$ and $\mathrm{CF}$ enzymes, we compared light-driven activities of $\mathrm{C}$ and $\mathrm{CF}$ in intact and solubilized thylakoid membranes. Solubilization of the membrane reduced the activity of the CF enzyme by $80 \%$ but did not affect that of C (Figure 4c).

Dependence of Activity on lonic Strength. Because Fd interacts electrostatically with redox partners, ${ }^{27}$ we examined the effect of the ionic strength $(I)$ on the interaction between $\mathrm{Fd}$ and the enzymes $\mathrm{C}$ and CF. The activity of $\mathrm{C}$ showed a marked bell-shaped dependence on $I$, reaching a maximum at $I$ $=60 \mathrm{mM}$, whereas CF was largely unaffected by $I$ (Figure 5a). To test whether fusion with $\mathrm{Fd}$ enabled $\mathrm{CF}$ to acquire more electrons from photosystem I even in the presence of a competing sink, we examined the effect of $I$ on the light-driven activity in the presence of FNR and the acceptor $\mathrm{NADP}^{+}$. FNR and $\mathrm{NADP}^{+}$competed strongly with $\mathrm{C}$ and $\mathrm{CF}$ for reduced ferredoxin, as the catalytic activities of both enzymes were reduced in their presence. However, CF retained more activity (up to $57 \%(78 \%)$ of its peak activity in the presence (absence) of soluble ferredoxin, respectively) than $\mathrm{C}$ ( $18 \%$ of maximal or less) under these conditions (Figure $5 b$ ).

In Vivo Activity of C and CF. Because in vitro activities of $\mathrm{C}$ and CF depended greatly on assay conditions, we examined the in vivo activity of both chloroplast-targeted proteins by quantifying reaction products extracted directly from tobacco. The aldoxime produced by ER-localized CYP79A1 was previously shown to undergo glycosylation in tobacco plants. ${ }^{28}$ To test whether chloroplast-produced $p$-hydrophenylacetaldoxime was glycosylated in our transient transfection experiments, we treated extracts of infiltrated leaves with a commercial $\beta$ glucosidase mixture and observed the release of large amounts of $p$-hydroxyphenylacetaldoxime (Supporting Figure S4).

We also synthesized the glucoside $p$-glycosyloxyphenylacetaldoxime (see Supporting Information, Materials and Methods), which allowed us to quantify both free and 

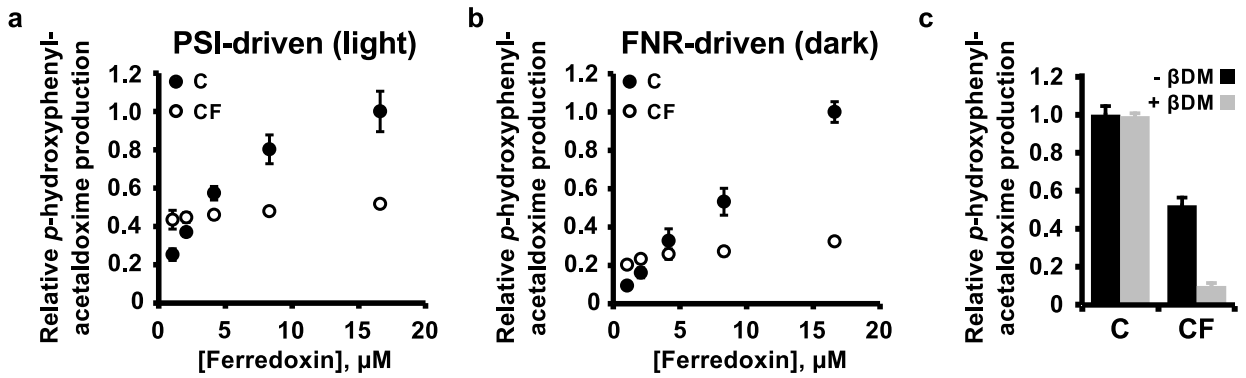

Figure 4. Effects of soluble ferredoxin and detergent on in vitro enzyme activity of $\mathrm{C}$ and CF proteins. (a) Light-dependent enzyme activity of thylakoids harboring either $\mathrm{C}$ or $\mathrm{CF}$, driven by electron transfer from soluble or fused ferredoxin reduced by photosystem I. (b) Enzyme activity of dark-incubated thylakoids harboring $\mathrm{C}$ or $\mathrm{CF}$, driven by electron transfer from soluble or fused ferredoxin reduced by $0.6 \mu \mathrm{M}$ FNR in the presence of $0.5 \mathrm{mM}$ NADPH. (c) Light-dependent activity of intact $(-\beta \mathrm{DM})$ or solubilized $(+\beta \mathrm{DM})$ thylakoids harboring $\mathrm{C}$ or $\mathrm{CF}$ in the presence of $8.3 \mu \mathrm{M}$ soluble Fd. Experimental values are means of four technical repeats each, normalized to relative protein levels determined by immunoblot, with error bars showing $\pm \mathrm{SD}$. $\beta \mathrm{DM}, n$-dodecyl $\beta$-D-maltoside.
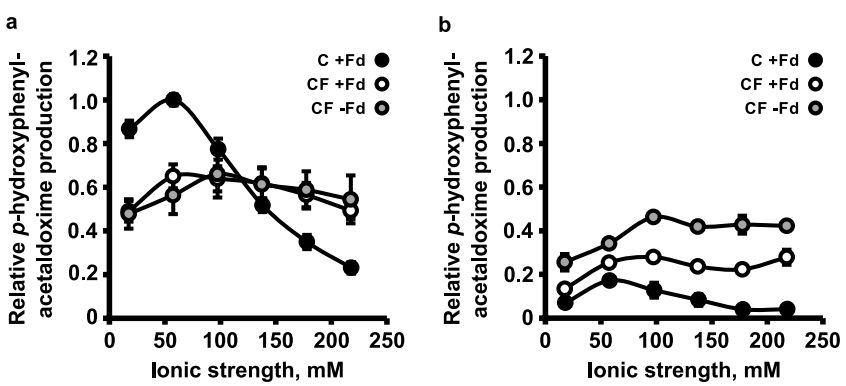

Figure 5. Effect of ionic strength on light-driven enzyme activity of tobacco thylakoids containing C (+soluble Fd) or CF ( \pm soluble Fd). (a) Dependence of $\mathrm{C}$ and $\mathrm{CF}$ activity on ionic strength, adjusted with $\mathrm{NaCl}$. (b) Dependence on ionic strength of $\mathrm{C}$ and $\mathrm{CF}$ activity measured in the presence of $0.6 \mu \mathrm{M}$ FNR and $1.63 \mathrm{mM} \mathrm{NADP}^{+}$acting as a competing electron sink for reduced $\mathrm{Fd}$. Data are plotted relative to the maximum activity of $\mathrm{C}$ obtained in a. Curves show mean activities from (a) four or (b) two technical replicates, normalized to the relative levels of $\mathrm{C}$ and $\mathrm{CF}$ proteins. Error bars indicate \pm SD.

glycosylated aldoxime using LC-MS/MS. We found amounts of p-glucosyloxyphenylacetaldoxime to be linearly correlated with, and on average 100-fold higher (w/w) than, those of $p$ hydroxyphenylacetaldoxime (Supporting Figure S5). To ensure that differences in the average metabolite levels detected in Cand CF-expressing plants were not caused by differences in expression of $\mathrm{C}$ and $\mathrm{CF}$ enzymes, we also quantified the relative amounts of both proteins in total protein extracts from infiltrated leaves by immunoblotting and found average $\mathrm{CF}$ expression to be $40 \%$ that of C (Supporting Figure S6). The expression-normalized production of free and glycosylated $p$ hydroxyphenylacetaldoxime showed that the specific activity of $\mathrm{CF}$ is $50 \%$ higher than that of $\mathrm{C}$ (Figure 6).

\section{DISCUSSION}

This study reports the light-driven activity of three fusions between CYP79A1 and Fd and confirms the establishment of functional electron transfer from photosystem $\mathrm{I}$ to the heme domain in the CF enzyme. We expected the $\mathrm{C}^{\Delta} \mathrm{F}$ and $\mathrm{FC}^{\Delta}$ fusions to be soluble proteins, but both associated with thylakoid membranes, possibly via hydrophobic interactions with the F-G loop of CYP79A1 ${ }^{25}$ and/or electrostatic Arg and Lys interactions with membrane phospho- or sulfolipids. ${ }^{29,30}$ The $\mathrm{C}^{\Delta} \mathrm{F}$ fusion was barely detectable in isolated chloroplasts, but readily so in thylakoid membrane preparations (Figure 2). The $\mathrm{FC}^{\Delta}$ fusion protein showed the highest expression in both chloroplast and thylakoid preparations, but both it and $\mathrm{C}^{\Delta} \mathrm{F}$ gave rise to additional immunospecific bands below the main one (Figure $2 b$ and Supporting Figure S2a). Neither C nor CF, which include the full-length CYP79A1 sequence, appeared
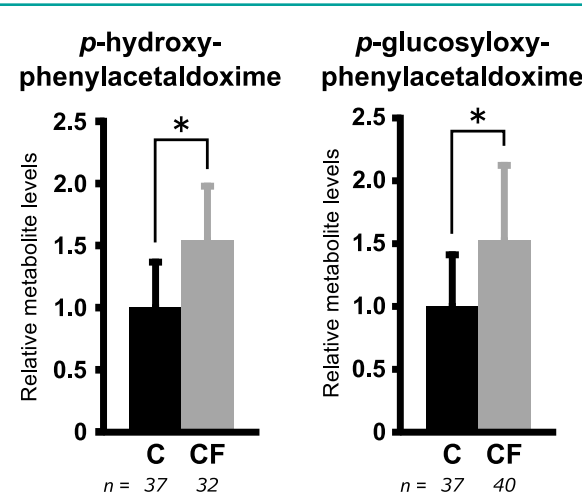

Figure 6. Relative amounts of $p$-hydroxyphenylacetaldoxime and $p$ glucosyloxyphenylacetaldoxime extracted from $N$. benthamiana leaves 5 days post infiltration with Agrobacterium strains bearing plasmids encoding either $\mathrm{C}$ or $\mathrm{CF}$, as quantified by LC-MS/MS and normalized to relative protein expression as determined by immunoblot. Equal numbers of leaf samples were analyzed for $p$-hydroxyphenylacetaldoxime and $p$-glucosyloxyphenylacetaldoxime in C $(n=37)$ and CF $(n=$ 40 ), but $p$-hydroxyphenylacetaldoxime was below the level of detection in some CF samples. Error bars \pm SD. *Statistical significance $(p<0.05)$ according to two-tailed unpaired $t$-tests.

susceptible to proteolysis and produced single bands at the expected positions on immunoblots (62 and $73 \mathrm{kDa}$ ). We conclude that truncation of CYP79A1 increases its susceptibility to chloroplast proteases, and this might explain the reduced activities of $\mathrm{C}^{\Delta} \mathrm{F}$ and $\mathrm{FC}^{\Delta}$. Other possible explanations are poor interaction between $\mathrm{Fd}$ and $\mathrm{P} 450$ domains, aberrant incorporation of heme or $2 \mathrm{Fe}-2 \mathrm{~S}$ clusters into the truncated 
proteins, or poor interaction with the PsaC, $-\mathrm{D}$, and $-\mathrm{E}$ subunits of photosystem I, which would limit electron transfer from its $\mathrm{F}_{\mathrm{B}}[4 \mathrm{Fe}-4 \mathrm{~S}]$ cluster (see Supporting Figure S1). ${ }^{31}$

Cytochrome $\mathrm{P} 450$ concentrations are commonly determined by inhibiting the dithionite-reduced enzyme with $\mathrm{CO}$ to yield a characteristic reduced-plus-CO vs reduced difference spectrum. ${ }^{32}$ We were unable to detect characteristic peaks at 450 or $420 \mathrm{~nm}$ because the high spectral background from pigments in the photosynthetic membrane swamps the weak signal expected from the P450:CO adduct (data not shown). Though CO spectra can also measure enzyme inactivation, the presence of other hemoproteins compromises their accuracy. ${ }^{32}$ Since thylakoid membranes contain many hemoproteins, in particular the Cytochrome $b_{6} \mathrm{f}$ complex, the method is unlikely to be accurate in this matrix. We instead measured activities in several independent thylakoid preparations and found them to be highly consistent overall, indicating that inactivation of the cytochromes does not cause major variation in this case.

The CF fusion was preferentially reduced by electrons transferred to its fused Fd domain by photosystem I , and only a minor fraction of its reducing power was derived from soluble Fd (Figure 7a). The most likely explanations for this preference are steric hindrance by the Fd domain limiting access of soluble Fd to the heme-proximal surface and much faster kinetics of electron transfer from photosystem I to the CF enzyme due to reduced dependence on diffusion. While our evidence does not

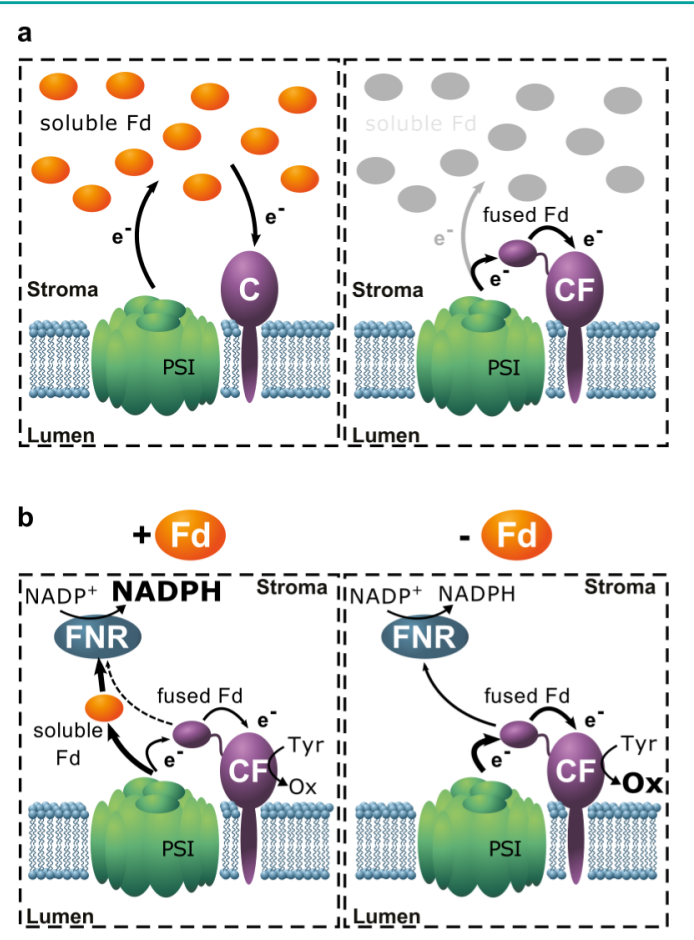

Figure 7. Electron transfer from photosystem I to CF independently of soluble $\mathrm{Fd}$ and competition between soluble and fused $\mathrm{Fd}$ for electrons in the presence of FNR. (a) Electron transfer chains involved during catalysis by $\mathrm{C}$ (left), where electrons flow from photosystem I (PSI) through the soluble Fd pool to the P450, and CF (right), where electrons flow from photosystem I via the Fd domain fused to the P450. (b) Electron flow toward FNR/NADP ${ }^{+}$vs $\mathrm{CF}$ in the presence of soluble Fd (left). When soluble Fd is absent (right), more electrons flow through the fused Fd domain of CF and consequently end up being used for hydroxylation of $\mathrm{Tyr}$; i.e., intramolecular electron transfer is faster than transfer to FNR. exclude other possibilities, a number of observations support this interpretation. First, the addition of high concentrations of soluble Fd resulted in only limited enhancement of CF activity (Figure $4 \mathrm{a}$ ). Second, the same trend was observed when FNR and NADPH were used to reduce $\mathrm{Fd}$, with activities of $\mathrm{C}$ and $\mathrm{CF}$ being equal at $\sim 4 \mu \mathrm{M}$ soluble $\mathrm{Fd}$ in both experiments (Figure $4 \mathrm{a}, \mathrm{b}$ ). Since FNR is a soluble enzyme, it is not affected by steric hindrance in the spatially highly organized environment of the thylakoid, which consists of $\sim 70 \%(\mathrm{~mol} / \mathrm{mol})$ protein $^{33}$ and allows only limited diffusion. ${ }^{34}$ Third, CF activity depends on colocalization with photosystem I in the thylakoid membrane, even when soluble $\mathrm{Fd}$ is present (Figure 4c). Solubilization of the membrane effectively causes its constituent proteins to be diluted, which is consistent with a decrease in activity of CF if it can only be reduced via direct interaction of its Fd domain with photosystem I. In contrast, the concentration of soluble $\mathrm{Fd}(8.3 \mu \mathrm{M})$ is unchanged by solubilization of the membrane, and therefore so also is the activity of the $\mathrm{C}$ protein. Finally, the higher specific activity of $\mathrm{CF}$ relative to unfused $\mathrm{C}$ under in vivo conditions (Figure 6) indicates that the differences observed between $\mathrm{C}$ and $\mathrm{CF}$ in vitro relate to the specific assay conditions used. Since the concentrations of $\mathrm{Fd}$ available to $\mathrm{CF}$ and $\mathrm{C}$ differ in principle, membrane colocalization of CF with photosystem I must compensate for its inability to interact with soluble Fd.

Unfused $\mathrm{C}$ protein showed typical saturation behavior as $\mathrm{Fd}$ concentrations were increased in light- but not in FNR-driven assays (Figure 4a,b). This difference probably results from slower reduction of Fd by FNR, because of thermodynamically uphill electron transfer from NADPH $(\mathrm{Em}=-320 \mathrm{mV})$ to $\mathrm{Fd}$ $(\mathrm{Em}=-433 \mathrm{mV}) .{ }^{35}$ From its saturation curve in light-driven assays we estimated the $K_{\mathrm{M}}$ of $\mathrm{C}$ for Fd to be $5.0 \pm 0.6 \mu \mathrm{M}$. For comparison, FNR - which consumes the majority of reduced Fd in vivo-has a $K_{\mathrm{M}}$ for $\mathrm{Fd}$ of $2.8 \mu \mathrm{M}$. $^{35}$ Such a high affinity toward an unnatural interaction partner can be rationalized by similarities between cytochrome P450 reductases (POR) and chloroplast Fd's. POR contains a small FMN-binding domain responsible for electron transfer to P450s, which is structurally homologous to the small electron carrier protein flavodoxin (Fld). ${ }^{36}$ Since Fld and Fd act interchangeably in some photosynthetic organisms, ${ }^{37}$ both carry highly negatively charged surfaces, and both interact electrostatically with photosystem I and FNR, ${ }^{38}$ their ability to support catalysis by many $\mathrm{P} 450 \mathrm{~s}^{8-11,39-41}$ is consistent with the hypothesis that these small electron carrier proteins evolved to interact with proteins through charge and surface shape complementarity in a relatively unspecific manner. ${ }^{42}$

The light-driven activity of $\mathrm{C}$ showed a bell-shaped dependence on ionic strength $(I)$ (Figure 5a). Similar $I$ dependences have been reported for Fd-dependent FNR activity and for reduction of plastocyanin by cytochrome c, and the increase in activity observed likely reflects increased dissociation rates due to suppression of nonspecific electrostatic interactions. $^{43-45}$ In contrast, the activity of the CF protein showed less dependence on ionic strength, probably because the covalent association of the interacting partners obviates the need for long-range electrostatic steering. The CF fusion is better able to compete with FNR for electrons (Figure 5b), but reduced CF activity in the presence of soluble Fd shows that the latter competes for interaction with photosystem I (Figure $7 b)$.

This work has important implications in relation to the choice of assay for assessment of P450-reductase fusions. Such 
enzymes are often evaluated in comparison with a stoichiometric reductase:P450 mixture, which can be misleading, since in vivo reductase:P450 ratios are often far from $1,{ }^{46}$ and the activities of fusion enzymes tend to increase linearly with concentration, while differing reductase: $\mathrm{P} 450$ ratios show nonlinear effects on rates. ${ }^{47}$ The choice of ionic strength is likewise important, because high ionic strengths would favor intramolecular electron transfer by less electrostatic-reliant fusion enzymes (Figure 5). In this study, we showed that our $\mathrm{CF}$ fusion can acquire more reducing power in the presence of a strong competing electron sink than unfused $\mathrm{C}$, which partly explains its increased in vivo activity. We should note, however, that in vivo concentrations of FNR and Fd (10-100 $\mu \mathrm{M}$ and 300-800 $\mu \mathrm{M}$, respectively, reported for chloroplasts and cyanobacteria $)^{48-50}$ would be difficult or impossible to replicate in vitro. Consequently, we stress the importance of backing up in vitro comparisons with in vivo experiments whenever possible.

Since light-driven cytochrome P450 pathways have key applications in live cell-culture production systems, we compared the in vivo activities of unfused $\mathrm{C}$ and the $\mathrm{CF}$ fusion. A preliminary time course analysis of $p$-hydroxyphenylacetaldoxime content in leaf extracts at $1-5$ days post infiltration showed levels peaking on day 3 , with no significant change on the fourth and fifth days (data not shown). The levels of $\mathrm{C}$ and $\mathrm{CF}$ proteins detected 5 days post infiltration indicated that the plants converted $p$-hydrophenylacetaldoxime to other compounds, and this was confirmed by enzymatic deglycosylation of extracted metabolites (Supporting Figure S4). We found free aldoxime to accumulate to 100 -fold lower levels than, and be linearly correlated with, its glucoside (Supporting Figure S6), which implies highly active glycosylation machinery for detoxification of foreign compounds, such as oximes. ${ }^{51,52}$ By quantifying both, we demonstrated that the catalytic activity of the fusion enzyme is superior to that of unfused CYP79A1 (Figure 6), thanks to its covalent association with $\mathrm{Fd}$, thus confirming that the thylakoid membrane possesses the plasticity required to allow interaction of heterologous enzymes with photosystem I.

This study represents the first report of enhanced lightdriven activity of a P450-reductase fusion in vivo. Improved light-driven hydrogenase activity through $\mathrm{Fd}$ fusion in vitro was previously demonstrated. ${ }^{53} \mathrm{We}$ did not explore the effect of linker length or composition in the present study, but both were recently found to influence electron transfer rates in fusions between the E. coli flavodoxin reductase and Fld. ${ }^{54}$ Though five residues were sufficient to support activity of a CYP11A1-adrenodoxin fusion, ${ }^{55}$ both studies found higher activity with longer linkers. ${ }^{54,55}$ Assembly of a complex between the model $\mathrm{P} 450_{\mathrm{cam}}$ and its reductase system (putidaredoxin and putidaredoxin reductase) by an alternative approach whereby they were fused with subunits of the PCNA trimer gave a 100fold rate increase, and this could be further increased by optimizing the linker, further demonstrating the rate enhancements achievable by proper linking of $\mathrm{P} 450$ and reductase. ${ }^{56,57}$ Consequently, we consider it likely that our reported CF fusion can be improved further by exploring alternate linker designs.

Conclusion. This study reports the in vitro and in vivo effects of introducing a P450-ferredoxin fusion protein into the thylakoid membranes of chloroplasts. The fusion CF could obtain electrons directly from photosystem I, and its activity was affected little by soluble Fd. The fusion enzyme also retained higher activity in the presence of competing electron sinks, probably because it is colocalized with photosystem I in the thylakoid membrane. Our P450-ferredoxin fusion approach thus enables direct coupling of photosynthetic electron transfer to P450s involved in desired biosynthetic pathways introduced into higher plant chloroplasts, algae, or cyanobacteria. To our knowledge, this is the first report of a fusion between a ferredoxin and a eukaryotic P450 involved in specialized metabolism. The CF fusion protein had a higher specific activity than unfused CYP79A1 in tobacco leaves, but was less abundant following transient expression. Minimal interaction between the fusion enzyme and soluble Fd makes the catalytic activity achieved by the fusion enzyme in vivo especially remarkable, since its fused $\mathrm{Fd}$ domain is present in substoichiometric amounts relative to free Fd. Evolution has tuned the distribution of photosynthetic reducing power to balance maximal biomass accumulation with the necessary redox regulation of metabolic processes. Thus, successful exploitation of photosynthetic organisms for light-driven production of high-value specialized metabolites or biofuels such as $\mathrm{H}_{2}$ will require strategies that modulate the distribution of reducing power. Our work indicates that $\mathrm{Fd}$ fusions constitute a transferable approach to channeling of photosynthetic reducing power into non-native pathways.

\section{ASSOCIATED CONTENT}

\section{S Supporting Information}

The Supporting Information is available free of charge on the ACS Publications website at DOI: 10.1021/acschembio.6b00190.

Materials and methods, Supporting Tables S1 and S2, Supporting Figures S1-S6 (PDF)

\section{AUTHOR INFORMATION}

\section{Corresponding Author}

*Tel.: +45 3533 3340. E-mail: peje@plen.ku.dk.

\section{Notes}

The authors declare no competing financial interest.

\section{ACKNOWLEDGMENTS}

The authors are indebted to G. T. Hanke (Queen Mary University of London) for valuable discussions and input regarding $\mathrm{Fd}$ and its interactions, and to $\mathrm{C}$. Crocoll for technical assistance. We thank L. M. Lassen (DTU) for help with illustrations. The research leading to these results received funding from the People Programme (Marie Curie Actions) of the European Union's Seventh Framework Program FP7/20072013/under REA Grant Agreement No. 317184. This material reflects only the views of the authors and the Union is not liable for any use that may be made of the information therein. The authors also acknowledge financial support from the VILLUM Center of Excellence in Plant Plasticity, the Center for Synthetic Biology (bioSYNergy) funded by the UCPH Excellence Programme for Interdisciplinary Research, the Innovation Fund Denmark (formerly the Danish Council for Strategic Research) Programme Commission on Strategic Growth Technologies (for "Plant Power: Light-Driven Synthesis of Complex Terpenoids Using Cytochromes P450" (Grant No. 12-131834), and the European Research Council [Advanced Grant "Light-driven Chemical Synthesis using Cytochrome P450s" (ERC-2012-ADG_20120314, Project No: 323034, Project Acronym: LightdrivenP450s)]. 


\section{REFERENCES}

(1) O'Connor, S. E. (2015) Engineering of Secondary Metabolism. Annu. Rev. Genet. 49, 71-94.

(2) Lassen, L. M., Nielsen, A. Z., Ziersen, B., Gnanasekaran, T., Møller, B. L., and Jensen, P. E. (2013) Redirecting Photosynthetic Electron Flow into Light-Driven Synthesis of Alternative Products Including High-Value Bioactive Natural Compounds. ACS Synth. Biol. 3,1 .

(3) Møller, B. L. (2014) Disruptive innovation: channeling photosynthetic electron flow into light-driven synthesis of high-value products. Synth. Biol. 1, 330.

(4) Schuler, M. A. (2015) P450s in Plants, Insects, and Their Fungal Pathogens, in Cytochrome P450, pp 409-449, Springer International Publishing, Cham, Switzerland.

(5) Gleadow, R. M., and Møller, B. L. (2014) Cyanogenic Glycosides: Synthesis, Physiology, and Phenotypic Plasticity. Annu. Rev. Plant Biol. 65, 155-185.

(6) Renault, H., Bassard, J., Hamberger, B., and Werck-Reichhart, D. (2014) Cytochrome P450-mediated metabolic engineering: current progress and future challenges. Curr. Opin. Plant Biol. 19, 27-34.

(7) Oliver, J. W. K., and Atsumi, S. (2014) Metabolic design for cyanobacterial chemical synthesis. Photosynth. Res. 120, 249-261.

(8) Nielsen, A. Z., Ziersen, B., Jensen, K., Lassen, L. M., Olsen, C. E., Møller, B. L., and Jensen, P. E. (2013) Redirecting Photosynthetic Reducing Power toward Bioactive Natural Product Synthesis. ACS Synth. Biol. 2, 308-315.

(9) Wlodarczyk, A., Gnanasekaran, T., Nielsen, A. Z., Zulu, N. N., Mellor, S. B., Luckner, M., Thøfner, J. F. B., Olsen, C. E., Mottawie, M. S., Burow, M., Pribil, M., Feussner, I., Møller, B. L., and Jensen, P. E. (2016) Metabolic engineering of light-driven cytochrome P450 dependent pathways into Synechocystis sp. PCC 6803. Metab. Eng. $33,1-11$.

(10) Gnanasekaran, T., Vavitsas, K., Andersen-Ranberg, J., Nielsen, A. Z., Olsen, C. E., Hamberger, B., and Jensen, P. E. (2015) Heterologous expression of the isopimaric acid pathway in Nicotiana benthamiana and the effect of $\mathrm{N}$-terminal modifications of the involved cytochrome P450 enzyme. J. Biol. Eng. 9, 24.

(11) Gnanasekaran, T., Karcher, D., Nielsen, A. Z., Martens, H. J., Ruf, S., Kroop, X., Olsen, C. E., Motawie, M. S., Pribil, M., Møller, B. L., Bock, R., and Jensen, P. E. (2016) Transfer of the cytochrome P450-dependent dhurrin pathway from Sorghum bicolor into Nicotiana tabacum chloroplasts for light-driven synthesis. J. Exp. Bot. 67, 2495.

(12) Jung, S. T., Lauchli, R., and Arnold, F. H. (2011) Cytochrome P450: Taming a wild type enzyme. Curr. Opin. Biotechnol. 22, 809817.

(13) Sadeghi, S. J., and Gilardi, G. (2013) Chimeric P450 enzymes: Activity of artificial redox fusions driven by different reductases for biotechnological applications. Biotechnol. Appl. Biochem. 60, 102-110.

(14) Englund, E., Andersen-Ranberg, J., Miao, R., Hamberger, B., and Lindberg, P. (2015) Metabolic Engineering of Synechocystis sp. PCC 6803 for Production of the Plant Diterpenoid Manoyl Oxide. ACS Synth. Biol. 4, 1270-8.

(15) Formighieri, C., and Melis, A. (2014) Regulation of $\beta$ phellandrene synthase gene expression, recombinant protein accumulation, and monoterpene hydrocarbons production in Synechocystis transformants. Planta 240, 309-324.

(16) Lindberg, P., Park, S., and Melis, A. (2010) Engineering a platform for photosynthetic isoprene production in cyanobacteria, using Synechocystis as the model organism. Metab. Eng. 12, 70-79.

(17) Angermayr, S. A., Gorchs Rovira, A., and Hellingwerf, K. J. (2015) Metabolic engineering of cyanobacteria for the synthesis of commodity products. Trends Biotechnol. 33, 352-361.

(18) Nielsen, A. Z., Mellor, S. B., Vavitsas, K., Wlodarczyk, A. J., Gnanasekaran, T., Perestrello Ramos $\mathrm{H}$ de Jesus, M., King, B. C., Bakowski, K., and Jensen, P. E. (2016) Extending the biosynthetic repertoires of cyanobacteria and chloroplasts. Plant J. Accepted Article, DOI: $10.1111 /$ tpj.13173.
(19) Jones, P. R., Møller, B. L., and Høj, P. B. (1999) The UDPglucose: p-hydroxymandelonitrile-O-glucosyltransferase that catalyzes the last step in synthesis of the cyanogenic glucoside dhurrin in Sorghum bicolor. J. Biol. Chem. 274, 35483-35491.

(20) Kahn, R. A., Bak, S., Svendsen, I., Halkier, B. A., and Møller, B. L. (1997) Isolation and reconstitution of cytochrome P450ox and in vitro reconstitution of the entire biosynthetic pathway of the cyanogenic glucoside dhurrin from sorghum. Plant Physiol. 115, 1661-70.

(21) Sibbesen, O., Koch, B., Halkier, B. a, and Møller, B. L. (1994) Isolation of the heme-thiolate enzyme cytochrome P-450TYR, which catalyzes the committed step in the biosynthesis of the cyanogenic glucoside dhurrin in Sorghum bicolor (L.) Moench. Proc. Natl. Acad. Sci. U. S. A. 91, 9740-9744.

(22) Sibbesen, O., Koch, B., Halkier, B. A., and Møller, B. L. (1995) Cytochrome P-450TYR is a multifunctional heme-thiolate enzyme catalyzing the conversion of L-tyrosine to p-hydroxyphenylacetaldehyde oxime in the biosynthesis of the cyanogenic glucoside dhurrin in Sorghum bicolor (L.) Moench. J. Biol. Chem. 270, 3506-3511.

(23) Clausen, M., Kannangara, R. M., Olsen, C. E., Blomstedt, C. K., Gleadow, R. M., Jørgensen, K., Bak, S., Motawie, M. S., and Møller, B. L. (2015) The bifurcation of the cyanogenic glucoside and glucosinolate biosynthetic pathways. Plant J. 84, 558-573.

(24) Kahn, R. a, Fahrendorf, T., Halkier, B. a, and Møller, B. L. (1999) Substrate specificity of the cytochrome P450 enzymes CYP79A1 and CYP71E1 involved in the biosynthesis of the cyanogenic glucoside dhurrin in Sorghum bicolor (L.) Moench. Arch. Biochem. Biophys. 363, 9-18.

(25) Jensen, K., Osmani, S. A., Hamann, T., Naur, P., and Møller, B. L. (2011) Homology modeling of the three membrane proteins of the dhurrin metabolon: Catalytic sites, membrane surface association and protein-protein interactions. Phytochemistry 72, 2113-2123.

(26) Reddy Chichili, V. P., Kumar, V., and Sivaraman, J. (2013) Linkers in the structural biology of protein-protein interactions. Protein Sci. 22, 153-167.

(27) Fukuyama, K. (2004) Structure and function of plant-type ferredoxins. Photosynth. Res. 81, 289-301.

(28) Bak, S., Olsen, C. E., Halkier, B. A., and Møller, B. L. (2000) Transgenic tobacco and Arabidopsis plants expressing the two multifunctional sorghum cytochrome P450 enzymes, CYP79A1 and CYP71E1, are cyanogenic and accumulate metabolites derived from intermediates in Dhurrin biosynthesis. Plant Physiol. 123, 1437-1448.

(29) Monk, B. C., Tomasiak, T. M., Keniya, M. V., Huschmann, F. U., Tyndall, J. D. a., O'Connell, J. D., Cannon, R. D., McDonald, J. G., Rodriguez, A., Finer-Moore, J. S., and Stroud, R. M. (2014) Architecture of a single membrane spanning cytochrome P450 suggests constraints that orient the catalytic domain relative to a bilayer. Proc. Natl. Acad. Sci. U. S. A. 111, 3865-3870.

(30) Headlam, M. J., Wilce, M. C. J., and Tuckey, R. C. (2003) The F-G loop region of cytochrome P450scc (CYP11A1) interacts with the phospholipid membrane. Biochim. Biophys. Acta, Biomembr. 1617, 96108.

(31) Andersen, B., Koch, B., Scheller, H. V., Okkels, J. S., and Møller, B. L. (1990) Nearest Neighbour Analysis of the Photosystem I Subunits in Barley and Their Binding of Ferredoxin, in Current Research in Photosynthesis, pp 1631-1634, Springer, Netherlands, Dordrecht.

(32) Guengerich, F. P., Martin, M. V., Sohl, C. D., and Cheng, Q. (2009) Measurement of cytochrome P450 and NADPH-cytochrome P450 reductase. Nat. Protoc. 4, 1245-1251.

(33) Dekker, J. P., and Boekema, E. J. (2005) Supramolecular organization of thylakoid membrane proteins in green plants. Biochim. Biophys. Acta, Bioenerg. 1706, 12-39.

(34) Kirchhoff, H., Mukherjee, U., and Galla, H.-J. (2002) Molecular architecture of the thylakoid membrane: lipid diffusion space for plastoquinone. Biochemistry 41, 4872-4882.

(35) Hanke, G. T., Kimata-Ariga, Y., Taniguchi, I., and Hase, T. (2004) A post genomic characterization of Arabidopsis ferredoxins. Plant Physiol. 134, 255-264. 
(36) Paine, M. J. I., Scrutton, N. S., Munro, A. W., Gutierrez, A., Roberts, G. C. K., and Wolf, C. R. (2005) Electron transfer partners of cytochrome P450. Cytochrome P450 Struct. Mech. Biochem. Third ed., pp 115-148, DOI: 10.1007/0-387-27447-2 4.

(37) Zhao, Q., Modi, S., Smith, G., Paine, M., McDonagh, P. D., Wolf, C. R., Tew, D., Lian, L. Y., Roberts, G. C., and Driessen, H. P. (1999) Crystal structure of the FMN-binding domain of human cytochrome P450 reductase at 1.93 A resolution. Protein Sci. 8, 298306.

(38) Ullmann, G. M., Hauswald, M., Jensen, A., and Knapp, E. W. (2000) Structural alignment of ferredoxin and flavodoxin based on electrostatic potentials: implications for their interactions with photosystem I and ferredoxin-NADP reductase. Proteins: Struct., Funct., Genet. 38, 301-309.

(39) Jensen, K., Jensen, P. E., and Møller, B. L. (2011) Light-driven cytochrome p450 hydroxylations. ACS Chem. Biol. 6, 533-539.

(40) Jensen, K., Johnston, J. B., de Montellano, P. R. O., and Møller, B. L. (2012) Photosystem I from plants as a bacterial cytochrome P450 surrogate electron donor: terminal hydroxylation of branched hydrocarbon chains. Biotechnol. Lett. 34, 239-245.

(41) Goñi, G., Zöllner, A., Lisurek, M., Velázquez-Campoy, A., Pinto, S., Gómez-Moreno, C., Hannemann, F., Bernhardt, R., and Medina, M. (2009) Cyanobacterial electron carrier proteins as electron donors to CYP106A2 from Bacillus megaterium ATCC 13368. Biochim. Biophys. Acta, Proteins Proteomics 1794, 1635-1642.

(42) Crowley, P. B., and Carrondo, M. A. (2004) The Architecture of the Binding Site in Redox Protein Complexes: Implications for Fast Dissociation. Proteins: Struct., Funct., Genet. 55, 603-612.

(43) Hurley, J. K., Morales, R., Martínez-Júlvez, M., Brodie, T. B., Medina, M., Gómez-Moreno, C., and Tollin, G. (2002) Structurefunction relationships in Anabaena ferredoxin/ferredoxin: $\operatorname{NADP}(+)$ reductase electron transfer: insights from site-directed mutagenesis, transient absorption spectroscopy and X-ray crystallography. Biochim. Biophys. Acta, Bioenerg. 1554, 5-21.

(44) Meyer, T. E., Zhao, Z. G., Cusanovich, M. a, and Tollin, G. (1993) Transient kinetics of electron transfer from a variety of c-type cytochromes to plastocyanin. Biochemistry 32, 4552-9.

(45) Kovalenko, I. B., Abaturova, A. M., Riznichenko, G. Y., and Rubin, A. B. (2011) Computer simulation of interaction of photosystem 1 with plastocyanin and ferredoxin. BioSystems 103, $180-7$.

(46) Estabrook, R. W., Franklin, M. R., Cohen, B., Shigamatzu, A., and Hildebrandt, A. G. (1971) Biochemical and genetic factors influencing drug metabolism. Influence of hepatic microsomal mixed function oxidation reactions on cellular metabolic control. Metab., Clin. Exp. 20, 187-99.

(47) Sibbesen, O., De Voss, J. J., and Ortiz de Montellano, P. R. (1996) Putidaredoxin Reductase-Putidaredoxin-Cytochrome P450 cam Triple. J. Biol. Chem. 271, 22462-22469.

(48) Okutani, S., Hanke, G. T., Satomi, Y., Takao, T., Kurisu, G., Suzuki, A., and Hase, T. (2005) Three maize leaf ferredoxin:NADPH oxidoreductases vary in subchloroplast location, expression, and interaction with ferredoxin. Plant Physiol. 139, 1451-1459.

(49) Moal, G., and Lagoutte, B. (2012) Photo-induced electron transfer from photosystem $\mathrm{i}$ to NADP+: Characterization and tentative simulation of the in vivo environment. Biochim. Biophys. Acta, Bioenerg. 1817, 1635-1645.

(50) Antal, T. K., Kovalenko, I. B., Rubin, A. B., and Tyystjärvi, E. (2013) Photosynthesis-related quantities for education and modeling. Photosynth. Res. 117, 1-30.

(51) Riechers, D. E., Kreuz, K., and Zhang, Q. (2010) Detoxification without intoxication: herbicide safeners activate plant defense gene expression. Plant Physiol. 153, 3-13.

(52) Møller, B. L. (2010) Plant science. Dynamic metabolons. Science 330, 1328-9.

(53) Yacoby, I., Pochekailov, S., Toporik, H., Ghirardi, M. L., King, P. W., and Zhang, S. (2011) Photosynthetic electron partitioning between $[\mathrm{FeFe}]$-hydrogenase and ferredoxin:NADP+-oxidoreductase (FNR) enzymes in vitro. Proc. Natl. Acad. Sci. U. S. A. 108, 9396-401.
(54) Bakkes, P. J., Biemann, S., Bokel, A., Eickholt, M., Girhard, M., and Urlacher, V. B. (2015) Design and improvement of artificial redox modules by molecular fusion of flavodoxin and flavodoxin reductase from Escherichia coli. Sci. Rep. 5, 12158.

(55) Strushkevich, N., MacKenzie, F., Cherkesova, T., Grabovec, I., Usanov, S., and Park, H.-W. (2011) Structural basis for pregnenolone biosynthesis by the mitochondrial monooxygenase system. Proc. Natl. Acad. Sci. U. S. A. 108, 10139-10143.

(56) Hirakawa, H., and Nagamune, T. (2010) Molecular Assembly of P450 with Ferredoxin and Ferredoxin Reductase by Fusion to PCNA. ChemBioChem 11, 1517-1520.

(57) Haga, T., Hirakawa, H., and Nagamune, T. (2013) Fine tuning of spatial arrangement of enzymes in a PCNA-mediated multienzyme complex using a rigid poly-L-proline linker. PLoS One 8, e75114. 